\title{
MORPHOLOGY AND MORPHOGENESIS OF NEPHROPATHY IN PSORIASIS VULGARIS
}

\section{Volos L. I.}

\section{INTRODUCTION}

Psoriasis is one of the most common dermatoses and accounts from $12 \%$ up to $15 \%$ of all skin diseases. It is not only a cosmetic defect, but a severe dermatosis with various clinical manifestations of more than 40 forms. Today's level of knowledge allows us to define psoriasis as a systemic disease, which includes a complex of pathogenetic units, such as metabolic, immune, nerve-endocrine, genetic and other disorders ${ }^{1}$. Visceral pathology, which is manifested in patients with psoriasis, has been attracting great attention of many researchers for many years ${ }^{2}$.

One of the central places in the visceral lesions in psoriasis is the pathology of the kidneys - "psoriatic nephropathy". In a monograph on nephrology, in the section of skin diseases and renal pathology, R.J. Glassosk ${ }^{3}$ states that often skin lesions can be dominant in kidney diseases, and, conversely, renal lesions can be manifestations of a general syndrome and be very weak, sometimes found circumstantially. The attention of dermatologists has been long and repeatedly drawn to the presence of renal pathology in patients with psoriasis.

It is thought that the controversy of data about the frequency and severity of damage to the kidneys in psoriasis is due to the fact that the authors make generalizations based on a diverse contingent of patients. In some cases it is meant a mild form of psoriasis, such as limited infiltrative plaque, in others more severe, such as psoriatic erythroderma or arthropathic form. Nowadays, the descriptions of individual clinical observations of various types of glomerulopathies associated with psoriasis are constantly appearing in issues of psoriasis literature in different countries, including Ukraine ${ }^{4}$.

1 Шевченко Т.И., Шлопов В.Г. Патологическая анатомия псориатической болезни: Киев, УкрНТЕК, 2001. 358 с.

2 Akoglu H, Dede F, Akoglu G, Gonul II, Odabas A.R. Membranoproliferative glomerulonephritis associated with psoriasis vulgaris. Ren Fail. 2009; 31(9):858-61.

${ }^{3}$ Glassock R.J. Cutaneus Disease. In Textbook of Nephrology. Baltimore; Maryland, 1995. 1: 859-860.

${ }^{4}$ Шлопов В.Г., Шевченко Т.І., Волос Л.І. Морфологія і морфогенез нефропатії при псоріазі. Київ. Видавництво АМН України, 1999. 184 с. 
The appearance of new scientific publications about different clinical and morphological manifestations of kidney damage suggests that glomerulopathy in psoriasis is not homogeneous in morphology and pathogenesis. Various clinical and morphological variants of amyloidosis, glomerulonephritis of immune origin $^{5,6}$ nephroangiosclerosis ${ }^{7}$ are concidered to refer to "psoriatic nephropathy" $", 9$. Taking to consideration the combination of psoriasis and gout, this group also includes the so-called "renal metabolic pathology" in the form of gouty (urate) nephropathy ${ }^{10}$. Thus, psoriatic nephropathy is a wide heterogeneous group of renal lesions. In our opinion, the clarification of the etiology and features of the development of nephropathy in all cases is necessary not only from the theoretical, but also from the practical point of view. Different level in progress of the disease, the rate of development of chronic renal failure, the possibility of influencing some mechanisms of development at pathology make it possible to detect the clinical and morphological features of psoriatic nephropathy. Knowledge of nephropathy morphology will allow dermatologists and therapists to avoid possible complications when prescribing some modern medicines.

\section{Clinical and morphological aspects of skin and visceral pathology in psoriasis: current condition of the issue}

There are a few dermatoses that have an ancient and interesting history, such as psoriasis. The description of the clinical layout of psoriasis is known since Hippocrates and Celsus times. The history of dermatology clearly shows how physicians' assessment of the general condition of patients with psoriasis has been changed. Psoriasis is a recurrent systemic disease that belongs to the group of chronic dermatoses. During the special examination

5, Jiao Y., Xu H., Li H., Li X. Mesangial proliferative glomerulonephritis with or without IgA deposits: the morphological characters in psoriasis vulgaris. Nephron Clin Pract. 2008. 108(3): 221-5.

${ }^{6,}$ Kaji T, Tsukada Y, Shimada, Miyamoto, Yoshida, Yano S., Naruse T. Memranous nephropathy associated with psoriasis vulgaris. Clin Nephrol. 1994. 4 (1): 63-4.

${ }^{7}$ Cai Y.I., Sich M., Beziai A, Kleppel MM, Gubler MC. Collagen distribution in focal and segmental glomerulosclerosis: an immunofluorescence and ultrastructural immunogold study. Brit. J. Pathol. 1996. 179(2): 188-196.

${ }^{8}$ Panasiuk N.N., Mukhin N.A., Varshavskiǐ V.A., Maksimov N.A. The clinicomorphological characteristics of psoriatic nephropathy. Ter Arkh 1990; 62(6): 99-103.

${ }^{9}$ Sakemi T., Hayashida R., Ikeda Y., Baba N., Nishihara G., Kohda H. Membranous glomerulonephropathy associated with psoriasis vulgaris. Nephron. 1996; 72(2): 351-2.

${ }^{10}$ Nicolae I., Tampa M., Ene C.D., Mitran C.I. et al. Correlations between relatedpurine derivatives and renal disorders in patients with psoriasis vulgaris. Exp Ther Med. 2019 Feb; 17(2):1012-19. 
of the skin, it was found that pathomorphological changes in the dermal vessels in patients with psoriasis appear long before clinical manifestations in the epidermis and visceral pathology in psoriasis is due to the systemic vascular and microcirculatory disorders.

In a complex clinical examination in patients with psoriasis the damages to kidneys, liver, heart, gastrointestinal tract, respiratory tract, urogenital and immune systems have been found. In the skin of patients with psoriasis in areas of lesions there have been found inflammation, which corresponds to the image of the immune ${ }^{11}$. The pathological process involves all the structures of the dermis and epidermis. The increase in the severity of the clinical course of the disease or its exacerbation is accompanied by an increase in the defect of cellular immunity. Thus, in the vulgar form of psoriasis there has been revealed a moderate deficiency of T-lymphocytes, which are quantitive, observed in the active phase of the disease and disappear during remission. A notable decrease in the absolute and relative numbers of T-lymphocytes is observed in psoriatic arthritis.

Data on the status of humoral immunity in psoriatic disease are controversial. In most cases, they show an increased content of immunoglobulins (Ig) of various classes. Approximately in $45 \%$ of patients with psoriasis antibodies against IgA and IgG are detected. After treatment, the number and activity of auto-AT are likely to decrease. Such disorders accompany autoimmune diseases and indicate the presence of an autoimmune component in the pathogenesis of psoriasis ${ }^{12}$. The increase of immunoglobulins $\mathrm{A}$ and $\mathrm{E}$ is the most characteristic feature for patients with erythrodermic form of psoriasis. Immunoglobulins A, M, G are part of circulating immune complexes, which role in the pathogenesis of psoriasis is the main ${ }^{13}$. An increase in the CIC level is found in 50\% of patients with vulgar psoriasis, in $73 \%$ of patients with exudative forms of psoriasis and in all patients with psoriatic arthritis.

The role of antigen-antibody complexes in the development of nephropathy has attracted the attention of researchers, as kidneys are the prime target for immunocomplex damage. Filtration-promoting mechanisms

${ }^{11}$ Kurihara K., Fujiyama T., Phadungsaksawasdi P., Ito T, Tokura Y. Significance of IL-17A-producing $\mathrm{CD}^{+} \mathrm{CD} 103^{+}$skin resident memory $\mathrm{T}$ cells in psoriasis lesion and their possible relationship to clinical course. J Dermatol Sci. 2019 Jul; 95(1): 21-7.

${ }^{12}$ Zheng J., Liu N., Wang Y., Chen F., Ding Y. Pemphigus vulgaris with psoriasis vulgaris successfully treated with methotrexate and low-dose methylprednisolone. Dermatol Ther. 2020 Jan; 33(1): e13165.

13 Owczarczyk Saczonek A., Krajewska-Włodarczyk M., Kasprowicz-Furmańczyk M., Placek W. Immunological Memory of Psoriatic Lesions. Int J Mol Sci. 2020 Jan 17; 21(2). pii: E625. 
provide the delay and the deposition of circulating immune complexes (CICs). This is prior due to the presence of the receptors on the epithelial and mesangial cells for the $\mathrm{C} 3$ component of the compliment and $\mathrm{F}-\mathrm{c}-$ the $\mathrm{IgC}$ fragment; the deposition of immune complexes in the glomerulis of the kidney, according to some authors, is connected with the presence in their composition of antigens that are similar to the antigens of the glomerular basement membrane, which cause cross-reactivity to these antigens.

Histological examination of the renal biopsy revealed the presence of severe extra- and intracapillary proliferative glomerulonephritis. Immunofluorescence examination of kidney biopsies revealed the deposition of Ig A not only in the mesangium, due to electron microscopy the deposition of massive electron-dense deposits was revealed in the mesangial and subendothelial zones. In the observations of $\mathrm{H}$. Kida et al. ${ }^{14}$ vulgar common psoriasis was with mesangioproliferative glomerulonephritis and was characterized by moderate mesangial proliferation, unevenly thickening of the glomerular capillary walls, narrowing or occlusion of the capillary lumen, polymorphic and reactive epithelium proliferation of Bowman's capsule, fusing it with capillary loops in separate glomeruli. In the immunofluorescence study, glomerulonephritis was characterized by subendothelial, subepithelial, mesangial electron-dense deposits, bright luminescence of $\operatorname{IgG}, \operatorname{IgA}$, and $\mathrm{C} 3$ complimenting the component in the mesangium and along the walls of the glomerular capillaries. All these confirms the immune complex mechanism of the damage. Kaji T. et al. ${ }^{15}$ first described an observation in which membranous nephropathy had been manifested in a 51-year-old man wiho had been suffering from psoriasis vulgaris for 10 years. In the kidney biopsy, granular IgG deposits along glomerular capillary walls had been detected by immunofluorescence. By electron microscopically it had been determined the fourth stage of the damage, which was characterized by uneven deposition of deposits and unequal thickness of the basement membrane. The use of prednisolone led to a decrease in the concentration of protein in urine and a significant improvement in the skin manifestations of the disease. The authors believe that the basis of pathogenesis of membranous nephropathy in the case was an antigen-antibody mechanism.

Panasyuk N.N. et al. ${ }^{16}$ has studied the nature of nephropathy in psoriatic disease and analyzed 34 clinical observations of psoriasis with urinary

\footnotetext{
${ }^{14}$ Kida H., Asamoto T., Abe T., Tomosugi N., Hattori N. Psoriasis vulgaris associated with mesangiocapillary glomerulonephritis. Clin. Nephrol. 1985. 23 (5): 255-7.

${ }^{15}$ Kaji T., Tsukada Y., Shimada, Miyamoto, Yoshida, Yano S., Naruse T. Memranous nephropathy associated with psoriasis vulgaris. Clin Nephrol. 1994. 42 (1): 63-4.

16 Panasiuk N.N., Mukhin N.A., Varshavskiǐ V.A., Maksimov N.A. The clinicomorphological characteristics of psoriatic nephropathy. Ter Arkh 1990; 62(6): 99-103.
} 
syndrome and performed clinical and morphological comparisons. In 27 observations, a chronic glomerulonephritis has been diagnosed, with amyloidosis morphologically confirmed in 7 patients. In clinical and morphological comparisons, basically all cases of glomerulonephritis and amyloidosis have been found in complicated, common forms of psoriasis. The combination of these features have been detected in 14 cases. The authors noted that psoriatic nephropathy may be represented by chronic glomerulonephritis with pronounced nephrotic syndrome, malignant arterial hypertension. The swellings were in the form of anasarka and it was difficult enough to undergo an active therapy, including hemosorption, high doses of glucocorticoids and cytostatics, symptomatic therapy, there was a rapid increase in signs of renal failure with the transition to terminal renal failure. Morphologically, in these observations, mesangio-proliferative glomerulonephritis was detected.

The problem undoubtedly deserves attention, due to the increase in complicated forms of dermatosis, which increases the frequency of damage to the internal organs, which are the main causes of death in patients. For many years, studies, that focuse on the study of kidney damage in psoriatic disease, have been single, and mainly descriptive, based on light-optical study of histological changes in the kidneys of psoriatic disease with the detection of kidney pathology, mainly in clinical morphology. And the patterns of kidney damage in patients with psoriasis remain unclear. Further study of the frequency, nature and morphogenesis of changes in kidneys, conducting clinical and morphological comparison of skin lesions and various nephropathies will reveal possible causes of kidney damage in psoriasis, the development and course of renal pathology, depending on the form of dermatosis, that will help to solve the issues of prevention of nephropathy in psoriasis, as well as identify ways to treat both dermatosis and associated organ pathology.

\section{Structural changes in the skin with psoriasis vulgaris}

On the previous phase of our present study, we examined 18 sectional observations of people, who suffered from psoriasis vulgaris (single plaques on extending surfaces of the elbow and / or knee joints) with prolonged remissions (from 2 to 10 years) and rare relapses. Hormonal drugs were not used in the complex therapy of this group of patients. The psoriatic nature of the skin lesion was based on a visual examination by professor of dermatology I.V. Svistunov. Subsequently, the diagnosis was confirmed on the basis of histological examination, as well as by kataanamnesis in the study of outpatient charts and case histories, which are kept in the archives of regional and the city dermatological and venereological dispensaries, district hospitals at the place of residence of the patients. 
The study was conducted in accordance to the basic bioethical provisions of the Council of Europe Convention on Human Rights and Biomedicine (04.04.1997), the World Health Association's Helsinki Declaration on Ethical Principles for Scientific Research with Human Participation (1964-2008), as well as the Order of the Ministry of Health of Ukraine No. 690 dated September 23, 2009.

On the external examination of the skin of people who have suffered from limited psoriasis, the papular, symmetrically located rash of various shapes and sizes has drawn attention. The rash was localized on the skin of the extending surfaces of the upper and lower extremities in the form of multiple single small papules, which in some areas merged with each other and formed plaques. The contours of the rash are quite clear. The colour of the papules is mainly cyanotic, gray-pink or silver-white with a strong plate flaking of their surface. The phenomenon of stearin spots and psoriatic film is revealed when the papular elements are crushed.

Complex histological, histochemical, immunohistochemical, ultrastructural, morphometric studies and polarization optical microscopy of the morphofunctional state of the skin and kidneys were performed in 18 observations of psoriasis vulgaris and compared with normal skin and kidneys (5 observations). The tissue pieces were fixed in $10 \%$ neutral formalin solution. Formalin-fixed pieces by standard method were poured into paraffin. Paraffin blocks on the rotary microtome MPS-2 made histological sections with a thickness of $5 \pm 1 \mu \mathrm{m}$. Paraffin sections were stained with hematoxylin and eosin, van Gizon, Vergoff, for the detection of sulfated and unsulfated glycosaminoglycans, the preparations were stained with toluidine blue ( $\mathrm{pH}=2.6$ and 5.3), neutral mucopolysaccharides were reacted with PAS-reaction. Amyloidosis was differentiated with hyalinosis by Congo-red staining and polarizing light microscopy.

For the part of serial histologic sections after deparaffinization by standard procedure, we used monoclonal antibodies labeled with peroxidase to surface antigens of the general population of $\mathrm{T}$ lymphocytes, B lymphocytes, CD4, CD8 and to actin. The quantitative polarization microscopy we used for measured the initial optical power of collagen fibers on paraffin sections immersed in Canadian balsam and the strength of their double refraction after the Ebner phenolic reaction, PAS- reaction, staining with toluidine blue, $\mathrm{pH}=5.3$. Phenolic index, indexes of neutral mucopolysaccharides and glycosaminoglycans by G.Scheuner, J. Hutschenreiter (1972) were calculated. The calibration charts determined the degree of maturity of the dermal collagen fibers and revealed the presence of secondary changes in them.

The morphometric studies were made by the basic principles summarized in the guidelines of GG Avtandilov (1980,1984). Electron microscopic and 
immunofluorescence study of the basement membrane of capillaries in the glomerulonephritis in patients with psoriasis was conducted in the pathohistological laboratory of the Hospital of St. Louis (Paris) "Laboratoire d'anatomie pathologique, Hôpital Saint-Louis, 1, avenue Claude Vellefaux, 75010 Paris, France" and we sincerely thank for the opportunity to do the research. We demonstrated a statistical analysis of the results of the study (analysis of variance, criterion $\chi 2$ ).

It is microscopically defined hyper- and parakeratosis. Among the thickened horny plates there are often stored cells of the granular layer with basophilic, oval nucleus and PAS-positive granularity of the cytoplasm. In the stratum corneum or directly beneath it, small accumulations of neutrophilic polymorphonuclear leukocytes can be found, which only in some papules form Munro microabscesses (Fig. 1).

Acanthosis is moderate mainly by increasing the number of cells of granular and spiny layers. The width of the prickly layer in most observations reached 17-18 rows of cells. The cells of the basal layer form one, and in some sections- two layers with single figures of mitosis. Epithelial growths and papillae of the dermis are not clearly expressed. The thickness of the epidermis in this group of patients was in average $287.53 \pm 17.09 \mu \mathrm{m}$. The average thickness of the normal epidermis is $42.63 \pm 5.68 \mu \mathrm{m}$.

The basement membrane of the dermo-epidermal junction is unevenly thickened, loosened, unevenly PAS-positive, intensely metachromatic when staining with toluidine blue at $\mathrm{pH}-5.3$, that indicates the accumulation both neutral mucopolysaccharides and depolymerysation of unsulfated glycosaminoglycans.

The degree of double refraction in the basement membrane and in the adjacent collagen fibers is significantly reduced, and in some places it is practically absent. In areas with double refraction, dichroism is preserved. The optical power of double refraction is 1.75 , and in sone places -1.69 (STD - 0.09-0.07, W - 4.0-5.4\%). In parallel with the decrease in the optical power of double refraction, there is a decrease in the phenolic index to 1.29-1.14 (STD - 0.03-0.04, W - 2.63-5\%) and an increase in the index of accumulation of neutral mucopolysaccharides to 2.52 ( STD - 0.11, $\mathrm{W}-4.4 \%$ ) and unsulfated glycosaminoglycans up to 2.20 (STD - 0.10, $\mathrm{W}-4.1 \%)$. These indicators are significantly different from the similar parameters obtained by us in the study of normal human skin. Thus, the optical power of double refraction of collagen fibers of the papillary layer of the dermis of normal skin is 3.32 (STD - 0.07, $\mathrm{W}-2.2 \%$ ), the phenolic index is equal to 2.24 (STD $-0.08, \mathrm{~W}-3.6 \%$ ), neutral mucopolysaccharide index 2.09 (STD - 0.09, W - 4.7\%), 
glycosaminoglycan index 1.77 (STD - 0.05, W - 3.2\%). These changes are manifestation of connective tissue disorganization and a reflection of the development of protein mesenchymal dystrophy, which varies in intensity from mucoid swelling to fibrinoid changes.

The dermal mesh layer is full-blooded, greatly thickened by swelling and swelling of collagen fibers (Fig. 2). In areas bordering the epidermis and around the vessels of the microhemocirculatory bed, the focal blurred metachromasia is revealed by staining with toluidine blue. Vessel walls are unevenly segmentally thickened, homogenized with areas of mucoid swelling. The most significant thickening of the basement membrane of the capillaries of the papillary layer of the dermis, which contains a large number of neutral mucopolysaccharides. The capillary endothelium is swollen, hypochromic. Venous vessels of the retina of the dermis are moderately ectated, full-blooded. The walls of large arteries are with hypertrophied muscular layer, sclerosis of the perivascular connective tissue.

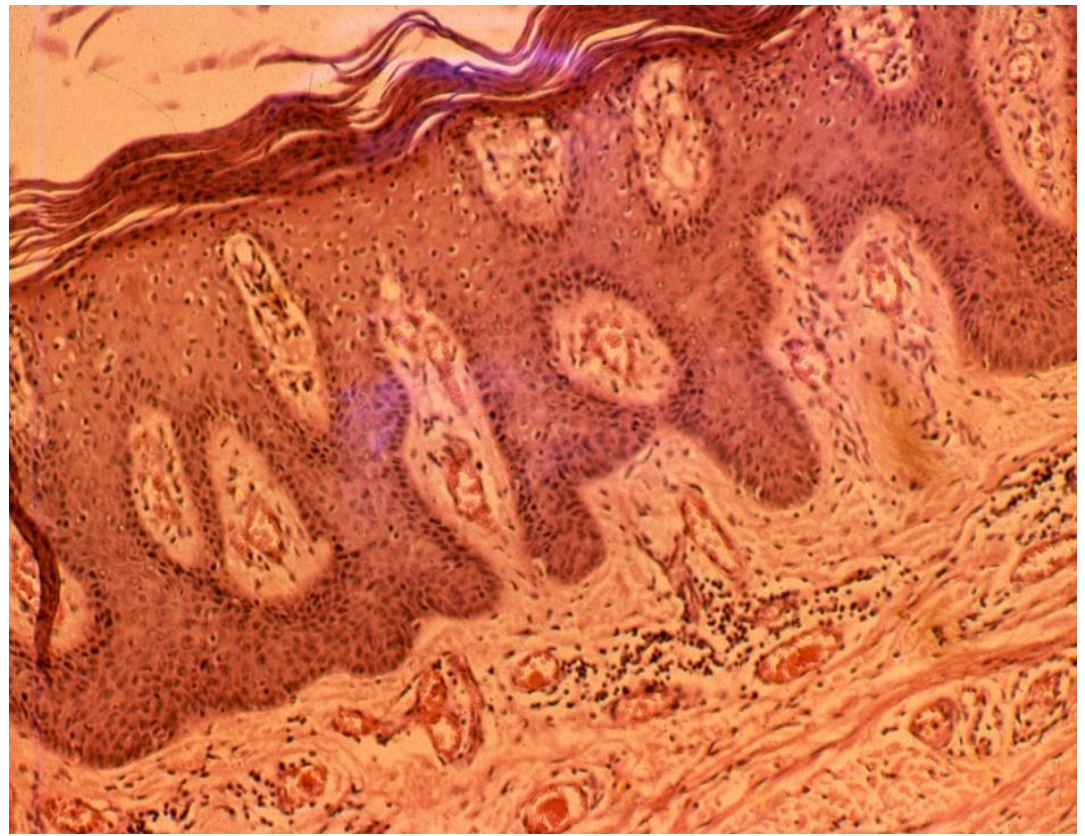

Fig. 1. Psoriasis Vulgaris. Sharply expressed hyperkeratosis, proliferative acanthosis, Munro micro abscesses, cellular infiltration of the dermis. Staining with hematoxylin and eosin, X400 


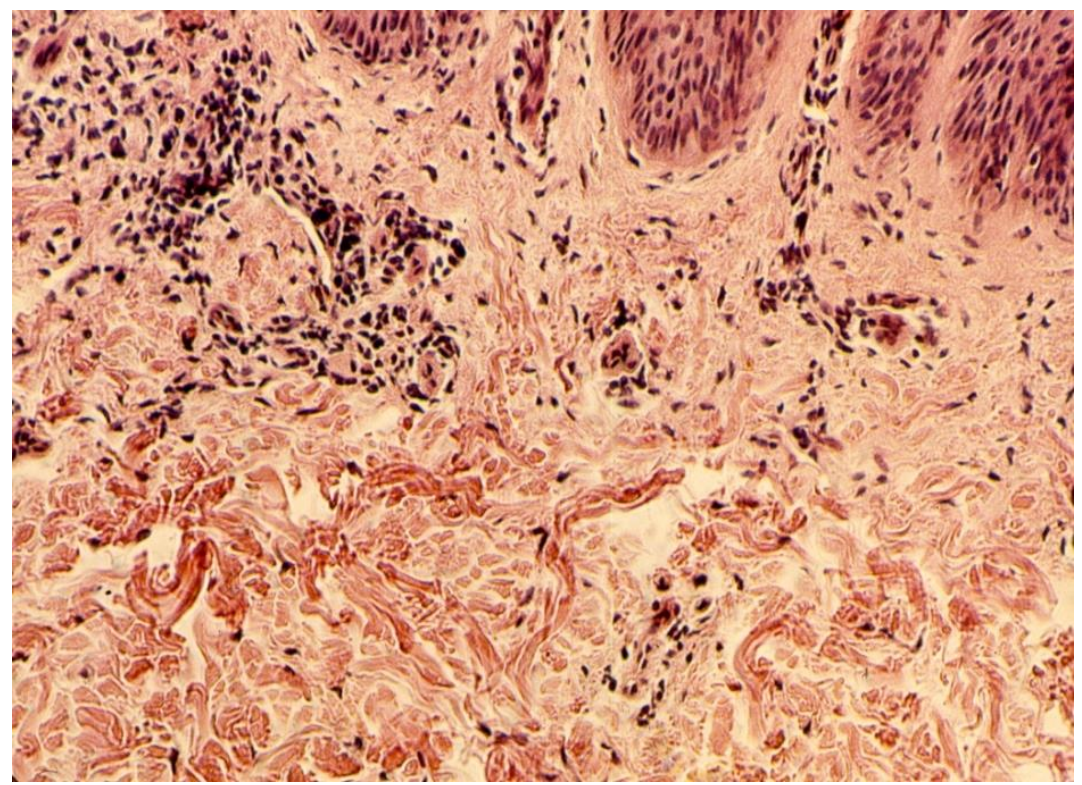

Fig. 2. Psoriasis Vulgaris. Dermal collagen fibers are swollen, fragmented, with areas of mucoid and fibrinoid changes.

There are small granulomas in the areas of destruction and they consist of macrophages and lymphocytes. Staining with hematoxylin and eosin, X400

The morphometric parameters of the vessels of the microhemocirculatory bed in conventional psoriasis were as follows: the specific volume of vessels $(\mathrm{Vv})$ was $0.195 \pm 0.013$; outer diameter of vessels $(\mathrm{Dv}, \mu \mathrm{m})-49,20 \pm 3,07$; the wall thickness of the vessels $(\mathrm{Hv}, \mu \mathrm{m})$ is $3.59 \pm 0.19$; $\mathrm{Dv} / \mathrm{Hv}-13.72$.The similar figures in the group of healthy individuals were as follows: specific vessel volume $(\mathrm{Vv})-0.042 \pm 0.004$; outer diameter of vessels $(\mathrm{Dv}, \mu \mathrm{m})-28,56 \pm 2,19$; The wall thickness of the vessels $(\mathrm{Hv}, \mu \mathrm{m})$ is $2.68 \pm 0.22$; $\mathrm{Dv} / \mathrm{Hv}-10.65$. Thus, in psoriatic papules and plaques compared with the control group, as well as in comparison with unchanged skin areas of the same patients, there is a significant increase in the volume of the vascular bed, the outer diameter and the thickness of the walls of the capillaries.

The collagen fibers of the dermis are swollen, fragmented with areas of mucoid and fibrinoid swelling and high level of neutral mucopolysaccharides. Small granulomas are located around the destruction sites, and consist of lymphocytes and macrophages, and sometimes only of 
macrophages. A small part of fibers is sclerosed, branched. Elastic fibers are unevenly thin, fragmented, in the deep parts of the dermis - without any foci. Polymorphocellular infiltrates are detected in both the mesh and papilla layers. Their specific volume is $5.51 \pm 0.8 \%$, which is 1.9 times higher than the specific volume of infiltrate, which is manifested in the apparently unchanged skin of the same patients. Dermal infiltrate is represented mainly by macrophages that form sites of nodules and solid layers, few mature lymphocytes with surface antigens of $\mathrm{T}$ cells ( $\mathrm{T}$-helper inducers), single small B-lymphocytes, single neutrophilic polymorphic polymorphisms, at different stages of degranulation.

\section{Structural changes in kidneys in psoriasis vulgaris}

Structural changes in kidneys in psoriasis vulgaris have been studied in 18 section cases. Puncture biopsies were examined in 2 patients with the usual form of psoriasis, which were accompanied by nephrotic syndromes of unclear origin.

In all section cases, irrespective of the limitation of the disease, localization and size (drop-shaped, small-papular, large-bulbous) of psoriatic rash, we have detected structural changes in both the glomerular apparatus of the kidneys and in the interstitium. The nature of morphological changes is diverse. These changes were inflammatory by nature and consistent with those described in various types of secondary chronic glomerulonephritis.

Psoriatic glomerulopathy with psoriasis vulgaris is characterized by irregular lesions of the vascular glomerular apparatus of the kidneys. Most glomerular structural changes are not observed. Glomerulopathy was focal in nature. In the affected glomeruli there is an uneven proliferation of mesangial cells in individual lobes, while in other areas vascular loops remain apparently unchanged in the light microscope.

In the adjacent glomeruli there has been observed a diffuse thickening of the wall of the peripheral capillary loops with the absence of pronounced cell proliferation, so, the pattern is characteristic of membranous glomerulopathy. There have also been glomeruli with predominantly mesangial diffuse cell proliferation, or with a combination of segmental membranous and segmental mesangial glomerulopathy (Fig. 3, 4 and 5).

During immunotyping it has been possible to reveal some regularities in the distribution of immune complexes from the nature of structural changes in the glomeruli. With mainly local deposition of immune complexes (IgA) (Fig. 6), there is a segmental lesion of the vascular loops of the glomerulus, for example, focal-segmental mesangioproliferative glomerulonephritis, and in the diffuse distribution of immune complexes - diffuse proliferation of mesangial and endothelial cells. 
In the study cases with psoriasis vulgaris there have dominated two main forms of glomerulopathy: mesangioproliferative and membranous glomerulonephritis. The minimal changes of the kidneys have been detected by us in one observation of a patient with vulgar psoriasis with a disease duration under 5 years.

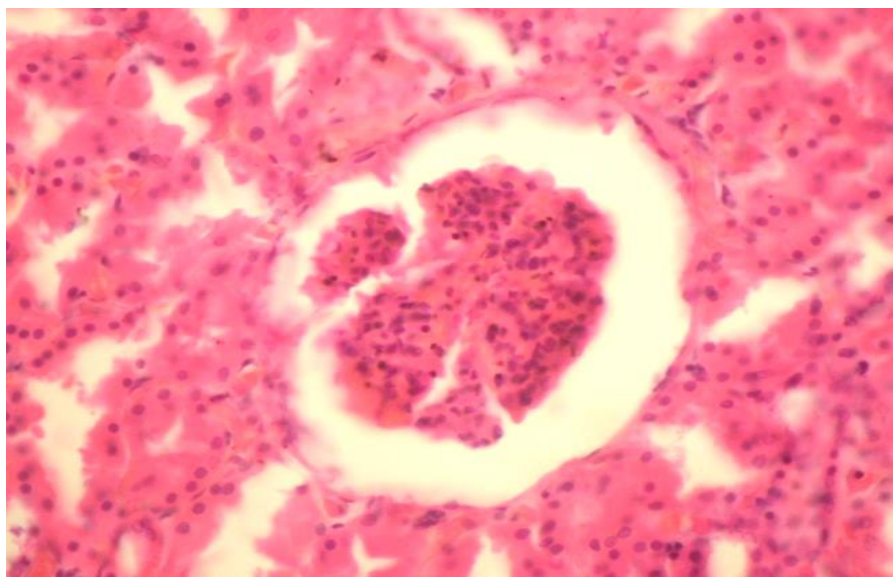

Fig. 3. Psoriasis Vulgaris. Mesangioproliferative glomerulonephritis: segmental proliferation of mesangial cells. Staining with hematoxylin and eosin, X400

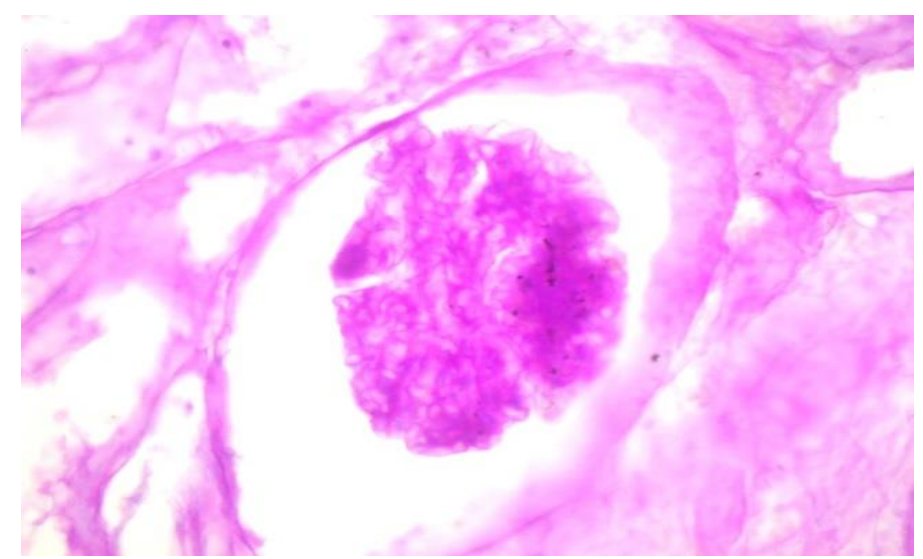

Fig. 4. Psoriasis Vulgaris. Mesangioproliferative glomerulonephritis: segmental proliferation of mesangial cells with segmental increases mesangial matrix. PAS-reaction, X400 


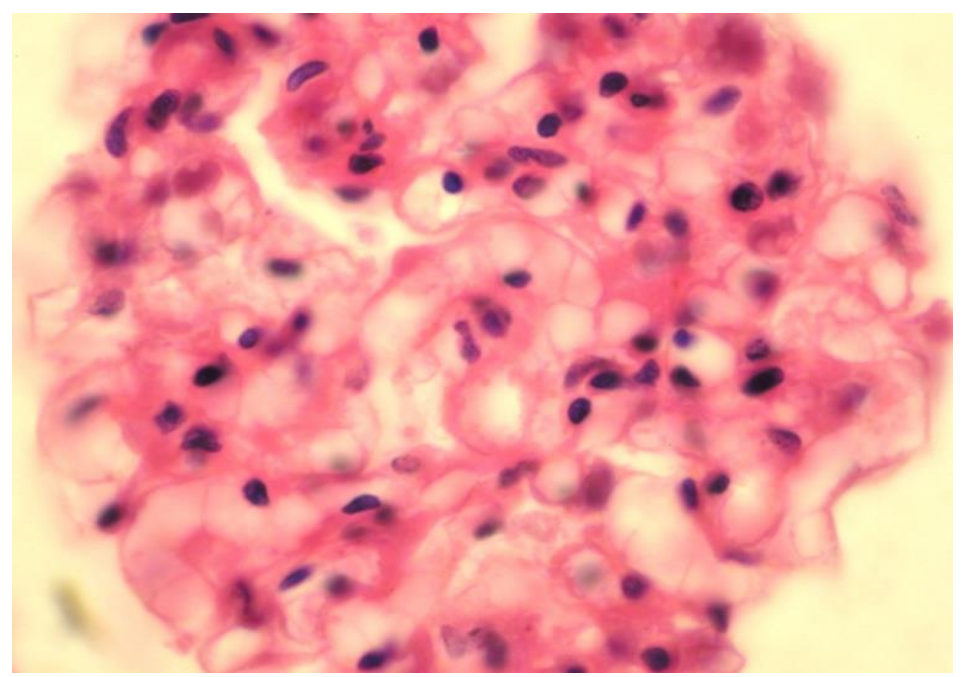

Fig. 5. Psoriasis Vulgaris. Membranous glomerulonephritis with focal thickening of the walls of the capillary glomerulus. Staining with hematoxylin and eosin, X1000 (immersion)

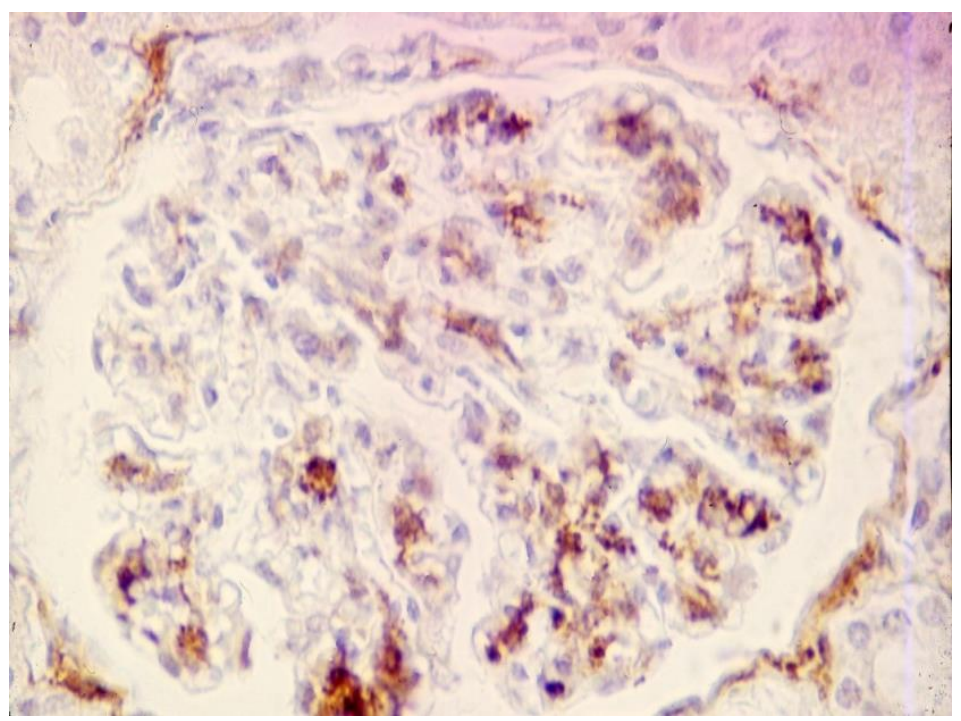

Fig. 6. Psoriasis Vulgaris. Mesangioproliferative glomerulonephritis: IgA depositions in the mesangial area, $\mathrm{X400}$ 
The extracapillary and tubulointerstitial components were in most cases weakly expressed. The extracapillary component was characterized by the fact that in 1 case of membranous and 2 cases of mesangioproliferative glomerulonephritis in some glomeruli it was revealed either protein exudate or accumulation of erythrocytes in the form of a crescent. The exceptions were two clinical observations of patients with a limited form of psoriasis and nephrotic syndrome, with a sufficiently defined extracapillary and interstitial components in the morphological examination of puncture kidney biopsies.

Following the collection of catanamnesis and the analysis of case histories of patients with vulgar limited psoriasis who were suddenly killed as a result of violent causes, it was found that none of the observations had indicated renal pathology. Thus, it could be assumed that the detected morphologically nephropathy was latent. However, four patients in urine tests had moderate proteinuria $(0.3-0.6 \mathrm{~g} / \mathrm{d})$. In two patients, it was combined with erythrocyturia with single leukocytes. In three cases, the results of urine tests are missing from the outpatient charts and medical records.

However, it should be noted that in three cases of mesangioproliferative and in one observation of membranous glomerulonephritis extracapillary component was detected. In addition, there was, although slightly notisable, focal interstitial component of psoriatic nephropathy. All that together indicated that the revealed morphologically nephropathy, at careful laboratory examination, could have had clinical manifestation.

This situation is confirmed by two cases of biopsies studied in patients with vulvar limited psoriasis with a vivid picture of nephropathy with nephrotic syndrome. Here is one of the observations.

Observation 1. Patient I., 32 years old, was admitted to the nephrological department of the regional clinical hospital with complaints about aching pain in the lumbar region, frequent urination, swelling of the face and lower extremities. He had beed suffering from limited vulgar psoriasis for five years after suffering a stressful situation. The last exacerbation was in April, and he was on treatment at the city skin and venereological dispensary. Urine changes were first discovered there: protein, leukocytes, erythrocytes. In May, swelling appeared on his face, waist, legs, which had persisted for three months, despite the symptomatic treatment. The patient had been offered an examination in the nephrology department to find out the cause of nephropathy.

In the clinic of nephrology with the radiological examination of the kidneys concrements were not found. Urine analysis: Qi. weight 1041, protein in daily diuresis was $14.8 \%$, no sugar, leukocytes -35 , erythrocytes 
leached - 50, hyaline cylinders - 7. Glomerular filtration $70.34 \mathrm{ml} / \mathrm{min}$. Blood test: erythrocytes - 4600 000; hemoglobin - 96; leukocytes - 11800, eos-2, neutr-74, lymphocytes - 16, monocytes - 7, ESR-43 mm / h. The residual blood nitrogen is $72 \mathrm{mg} \%$.

A detailed clinical examination revealed no concomitant pathology. In ECG examination diffuse changes of the heart muscle (diffuse cardiosclerosis) were revealed. In order to clarify the nephrological diagnosis, an operation had been performed in October - a puncture biopsy of the kidney by a half-open method.

The histological examination of biopsy material (histological analysis No. 55205) revealed 15 glomeruli in the preparation. Almost all of them were increased in volume. In some of them the vascular glomerulus occupies the entire cavity of the Bowman's capsule. They expressed focal proliferation of mesangium cells and capillary endothelium, protein masses were in the capsule cavity of some glomeruli. In stroma there were swelling and branching, poorly expressed lymphocytic infiltration. In two glomeruli there was focal thickening of the capsule with a small proliferation of epithelial cells. Conclusion (Prof. Shlopov VG): mesangioproliferative glomerulonephritis.

After intensive care, the patient's condition improved slightly, the edema on the face and torso disappeared, at the lower extremities- decreased, but there was not a complete remission from the kidneys. Urinary syndrome persisted for a long time. At the conclusion of the commission, he was given a disability status and discharged for further observation and treatment at the place of residence.

The interest to the present observation is that a patient with psoriasis vulgaris with a relatively short history of the disease (less than five years) has a pronounced clinical picture of visceral lesions, in particular, kidneys and heart; nephropathy (mesangioproliferative glomerulonephritis) with a pronounced pattern of nephrotic syndrome dominated the clinical picture of common infiltrative plaque psoriasis; there was a synchronicity of both clinical and morphological exacerbation of the inflammatory process in both skin and kidneys; the presence of a combination of intraglomerular and extraglomerular proliferative inflammatory process gives the basis to suggest a combined immune mechanism of development of glomerulonephritis in this patient, probably due to different types of immunoglobulins that are part of the immune complexes, which are important.

The high linear positive correlation between the presence of various types of chronic focal-segmental glomerulonephritis and psoriasis vulgaris (0.945) indicates a coincidence of the hyperproliferative process in the skin and the proliferative inflammatory process. 
What can be suggested about the possible pathogenetic mechanisms of glomerular changes in a group of patients with a limited form of psoriasis?

Firstly, that are the changes in the kidneys that are probably related to the nephrotoxic effect of the clinic.

Secondly, changes in the kidneys, that ar possibly due to the common pathogenetic mechanisms in the skin and kidneys.

In order to exclude the fact that changes in kidneys are related to the nephrotoxic effect of the therapy performed at the clinic, we analyzed the nature of the treatment, the timing and the duration of its use. It should be noted that in the group of patients with a limited form of psoriasis, nearly all patients, with the exception of 2 patients with nephrotic syndrome, received comprehensive treatment in combination with HBO, PUVA therapy. None of the patients received cyclosporine, the use of which had been described in cases of cyclosporine kidney in the literature. In addition, 5 out of 8 patients were undergoing long-term remission (from 2 up to 3 years).

In our study group of cases there have been revealed different types of glomerulopathies with a predominance of segmental proliferative inflammation in response to the deposition of immune complexes, whose composition is most commonly determined by $\operatorname{IgA}$.

\section{CONCLUSIONS}

Therefore, in our opinion, glomerulopathy, which has been found in patients with a psoriasis vulgaris, is most likely pathogenetically and morphogenetically associated with the underlying disease. In favor of this:

- focal and segmental lesions of basal membranes of skin and kidney vessels;

- predominantly proliferative character of inflammation;

- immunocomplex (mainly IgA) nature of inflammation;

- weak interstitial and extracapillary component.

The clinically long, latent course of psoriatic nephropathy is caused by the fact that the lesions of the kidneys are predominantly focal in nature and are represented mainly by two main morphological forms: membranous and mesangioproliferative glomerulonephritis, which are known to be well characterized.

\section{SUMMARY}

It is established that in all forms of psoriasis there is a structural pathology of the kidneys, defined as psoriatic nephropathy, which is one of the manifestations of the visceral symptom complex of psoriatic disease.

The structural features of psoriatic nephropathy at all stages of its development include its focal and segmental nature of the lesions of the 
basal membranes of the glomerular capillaries, low percentage of sclerosed glomeruli (no more than 3\% with respect to all affected glomeruli, and in the group of patients with $1 \%$ ), which indicates a benign, prolonged course of the inflammatory process and explains its latent clinical course in vulgar uncomplicated psoriasis.

Psoriatic nephropathy is represented by the following basic morphological forms: minimal changes of the kidneys, mesangioproliferative, membranous, membranoproliferative, diffuse fibroplastic glomerulonephritis, as well as amyloidosis.

The most characteristic type of glomerulopathy in all forms of psoriasis is focal-segmental mesangioproliferative glomerulitis ( $\operatorname{IgA}$ - nephropathy), which accounts $61.1 \%$ of all structural kidney pathological findings of autopsy material.

In psoriasis vulgaris, nephropathy is manifested as focal-segmental mesangioproliferative and membranous glomerulonephritis.

In the pathogenesis of psoriatic nephropathy, the most likely mechanism is the deposition of immune complexes and a direct cell-mediated cytotoxic response, which manifests in the kidneys and skin with morphologically identical angiopathy at the level of the microcirculatory bed, an increase in the level of Ig, in particular IgA.

\section{REFERENCES}

1. Шевченко Т.И., Шлопов В.Г. Патологическая анатомия псориатической болезни. Киев : УкрНТЕК, 2001. 358 с.

2. Akoglu H., Dede F., Akoglu G., Gonul II., Odabas A.R. Membranoproliferative glomerulonephritis associated with psoriasis vulgaris. Ren Fail. 2009; 31(9):858-61. doi: 10.3109/08860220903180582.

3. Glassock R.J. Cutaneus Disease. In Textbook of Nephrology. Baltimore; Maryland, 1995. 1: 859-860.

4. Шлопов В.Г., Шевченко Т.І., Волос Л.І. Морфологія і морфогенез нефропатії при псоріазі. Київ : Видавництво АМН України, 1999. 184 с.

5. Jiao Y., $\mathrm{Xu}$ H., $\mathrm{Li} \mathrm{H} ., \quad \mathrm{Li} X$. Mesangial proliferative glomerulonephritis with or without IgA deposits: the morphological characters in psoriasis vulgaris. Nephron Clin Pract. 2008; 108(3): 221-5. doi: 10.1159/000119716. Epub 2008 Mar 7.

6. Kaji T., Tsukada Y., Shimada, Miyamoto, Yoshida, Yano S., Naruse T. Memranous nephropathy associated with psoriasis vulgaris. Clin Nephrol. 1994. 42 (1): 63-4.

7. Cai Y.I., Sich M., Beziai A., Kleppel M.M., Gubler M.C.. Collagen distribution in focal and segmental glomerulosclerosis: an immunefluorescence and ultrastructural immunogold study. Brit. J. Pathol. 1996. 179 (2): 188-196. 
8. Panasiuk N.N., Mukhin N.A., Varshavskiı̌ V.A., Maksimov N.A. The clinico-morphological characteristics of psoriatic nephropathy. Ter Arkh 1990; 62(6): 99-103. PMID: 2218939.

9. Sakemi T., Hayashida R., Ikeda Y., Baba N., Nishihara G., Kohda H. Membranous glomerulonephropathy associated with psoriasis vulgaris. Nephron. 1996; 72(2): 351-2. DOI: 10.1159/000188882.

10. Nicolae I, Tampa M., Ene C.D., Mitran C.I. et al. Correlations between related-purine derivatives and renal disorders in patients with psoriasis vulgaris. Exp Ther Med. 2019 Feb; 17(2):1012-19. doi: 10.3892/etm.2018.7053. Epub 2018 Dec 5.

11. Kurihara K., Fujiyama T., Phadungsaksawasdi P., Ito T., Tokura Y. Significance of IL-17A-producing $\mathrm{CD}^{+} \mathrm{CD} 103^{+}$skin resident memory $\mathrm{T}$ cells in psoriasis lesion and their possible relationship to clinical course. J Dermatol Sci. 2019 Jul; 95(1): 21-7. doi: 10.1016/j.jdermsci.2019.06.002.

12. Zheng J., Liu N., Wang Y., Chen F., Ding Y. Pemphigus vulgaris with psoriasis vulgaris successfully treated with methotrexate and low-dose methylprednisolone. Dermatol Ther. 2020 Jan; 33(1): e13165. doi: 10.1111/dth.13165. Epub 2019 Nov 15.

13. Owczarczyk Saczonek A., Krajewska-Włodarczyk M., KasprowiczFurmańczyk M, Placek W. Immunological Memory of Psoriatic Lesions. Int J Mol Sci. 2020 Jan 17; 21(2). pii: E625. doi: 10.3390/ijms21020625.

14. Kida H., Asamoto T., Abe T., Tomosugi N., Hattori N. Psoriasis vulgaris associated with mesangiocapillary glomerulonephritis. Clin. Nephrol. 1985. 23 (5): 255-7.

Information about author: Volos L. I., Doctor of Medical Sciences, Professor of the Department of Pathological Anatomy and Forensic Medicine Danylo Halytsky Lviv National Medical University 69, Pekarska str., Lviv, 79010, Ukraine 